\title{
Multi-reference Computational Method for De-novo Design, Optimization, and Repositioning of Pharmaceutical Compounds Illustrated by Identifying Multi-target SARS-CoV-2 Ligands
}

\author{
Vadim Alexandrov \\ Liquid Algo LLC https://orcid.org/0000-0002-0254-9799 \\ Alexander Kirpich \\ Georgia State University https://orcid.org/0000-0001-5486-0338 \\ Yuriy Gankin ( $\nabla$ yuriy.gankin@quantori.com ) \\ Quantori https://orcid.org/0000-0003-0046-1037
}

\section{Research Article}

Keywords: conformers, multi-reference, poly-conformational, in silico, ligand-based, structure-based, SARS COV-2, COVID-19, fingerprints, cheminformatics, similarity, virtual library, computational framework, validation

Posted Date: September 3rd, 2021

DOI: https://doi.org/10.21203/rs.3.rs-153954/v2

License: (c) (i) This work is licensed under a Creative Commons Attribution 4.0 International License.

Read Full License 


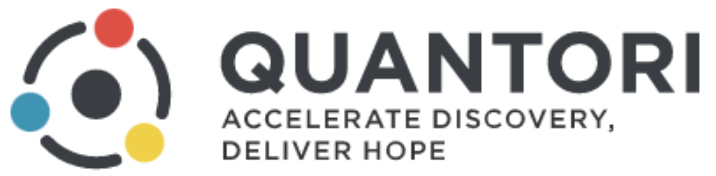

Dear Journal of Cheminformatics Editorial Team,

We appreciate your consideration of our manuscript entitled "Multi-reference computational method for de-novo design, optimization, and repositioning of pharmaceutical compounds illustrated by identifying multi-target SARS-CoV-2 ligands".

In this paper, we have proposed the multi-reference optimization approach in various in-silico drug discovery settings and illustrated its application and usefulness. We have also performed the validation of our approach for SARS-CoV-2 compounds and the corresponding results have been included in our manuscript. According to the proposed approach, each molecule is represented as an ensemble of flexible conformers that would choose the best possible conformation for each presented target-binding opportunity that can be applied in multiple settings. The aims were: a) to present a universal search framework for potential candidate compounds based on the comparison of multiple similarities between compounds' conformers and b) to identify candidate compounds that are simultaneously similar to each of the selected known reference compounds. Application of this approach to SARS-CoV-2 produced several antiviral drug candidates that are designed to protect against SARS-CoV-2 by multiple mechanisms simultaneously.

Since this is a time sensitive matter, we appreciate your expedited consideration and review of our manuscript for publication in the Journal of Cheminformatics. Please do not hesitate to contact me with any additional inquiries regarding this submission.

On behalf of all authors,

Yuriy Gankin, Ph.D., MBA

Quantori. Custom Software Solutions.

625 Massachusetts Avenue

Cambridge, MA 02139

United States

E-mail: yuriy.gankin@quantori.com

Phone: (781) 771-1978 
1 Multi-reference computational method for de-novo design, optimization, and

2 repositioning of pharmaceutical compounds illustrated by identifying multitarget SARS-CoV-2 ligands University, Atlanta, GA, United States of America

* Corresponding author 
In this work a novel computational multi-reference poly-conformational algorithm is presented for

21 design, optimization, and repositioning of pharmaceutical compounds. The algorithm searches for

22 candidates by comparing similarities between conformers of the same compound and identifies

23 target compounds whose conformers are simultaneously "close" to the conformers for each of the

24 compounds in a reference set. The reference compounds can have very different MoAs, which

25 directly and simultaneously shapes the properties of the target candidate compounds.

26 The algorithm functionality has been validated in silico by scoring ChEMBL drugs against FDA-

27 approved reference compounds which either had the highest predicted binding affinity to our

28 chosen SARS-COV-2 targets or confirmed to be inhibiting such targets in-vivo. All our top scoring

29 ChEMBL compounds also turned out to be either high-affinity ligands to the chosen targets (as 30 confirmed separately in other studies) or showing significant efficacy in-vivo against those 31 selected targets.

32 In addition to method validation in silico search for new compounds within two virtual libraries

33 from the Enamine database is presented. The library's virtual compounds have been compared to 34 the same set of reference drugs that we used for validation: Olaparib, Tadalafil, Ergotamine and 35 Remdesivir. The large reference set of four potential SARS-CoV-2 compounds have been selected, 36 since no drug has been identified to be $100 \%$ effective against the virus so far, possibly because 37 each candidate drug was targeting only one particular MoA. The goal here was to introduce 38 methodology for identifying potential candidate(s) that cover multiple MoA-s presented within a 39 set of reference compounds. 


\section{KEYWORDS}

43 conformers, multi-reference, poly-conformational, in silico, ligand-based, structure-based, SARS-

44 COV-2, COVID-19, fingerprints, cheminformatics, similarity, virtual library, computational 45 framework, validation.

48 Conformers as independent molecular entities. In real life, most compound molecules exist in multiple conformations (shapes) based on the surrounding environmental conditions. In particular, each 3D shape of a molecule dictates its biological activity and enables the molecule to fit into the

51 binding pockets of proteins. Often, distinctly different chemical compounds that have similar 52 shapes (and similar charge distributions along the molecular surface) have a potential to bind as 53 long as the ligand's partial charges are positioned in the binding pocket the same way (i.e., form 54 the same hydrogen bonds). Therefore, it is beneficial to compare the shapes and surface 55 distribution charges for target query and reference compounds on a conformer-by-conformer basis. 56 If one of the conformers of the query molecule matches one of the conformers (especially bound57 to-target) of the reference molecule, then there is a chance that the reference compound will also 58 exhibit similar binding properties to the same target.

59 Alignment-free 3D-similarity scoring. OpenEye Scientific Software Inc. pioneered an algorithm 60 and the corresponding tool ROCS ${ }^{13}$ for comparing shapes of molecules by overlaying and 61 measuring their molecular structures in silico and comparing differences between a query and 62 target molecule. ROCS identifies potentially active compounds by comparing their shapes. 
63 Moreover, the ROCS tool is competitive and often superior to structure-based approaches in virtual

64 screening ${ }^{14,15}$ both in terms of overall performance and consistency ${ }^{16}$. As a result, novel molecular

65 scaffolds have been identified by using ROCS against various targets which have been considered

66 very difficult to address computationally ${ }^{17}$.

67 Challenges with overlaying. The process of molecular shapes overlaying remains

68 computationally intensive and often is a bottleneck in the search process for similar molecules.

69 This remains despite the recent so-called PAPER implementation of ROCS on GPU ${ }^{18}$ and the 70 development of FastROCS ${ }^{19}$ for large (>1B) compound libraries. Recently, alternative methods

71 for overlaying have been introduced as a substitute for the ROCS approach. The alternative

72 overlaying is performed by comparing shape-based descriptors (a.k.a conformer-level 3D

73 fingerprints). An example of such an approach is ElectroShape implemented in the ODDT package

$74{ }^{20}$ and is based on the algorithm that incorporates shape, chirality, and electrostatics ${ }^{21,22}$, and

75 represents each conformer via a fixed-length vector of real-valued numbers. Similarly the E3FP

76 package ${ }^{23}$ also utilizes an alignment-invariant 3D representation of molecular conformers as a

77 fixed-length binary vector for each conformer. These fingerprint-based approaches allow to

78 calculate the similarity between two molecular shapes either as a Tanimoto distance (for binary

79 fingerprints) or Euclidean distance (for real-valued fingerprints) computations. Such computations

80 are orders of magnitude faster in comparison to alternative methods that require the actual

81 alignment of the two compared conformers. Even though the calculation of a shape-based

82 fingerprint for each conformer can be a rather computationally involved procedure, as soon as all

83 conformers for the virtual library are fingerprinted and stored in a database, the similarity search

84 for the query molecule in such a database is computationally quick. Therefore the computationally 
efficient method proposed here is expected to be very useful for finding candidate drugs for multitarget disease indications, ligand-based drug design, and drug repurposing applications.

Method applications for SARS-CoV-2 treatment compounds. The set of SARS-CoV-2 treatment compounds have been used for both method validation since, there are compounds that have been confirmed to be effective ${ }^{24}$ and for the search for new potential compounds based on the existing known set since no drug has been identified to be $100 \%$ effective against the virus, possibly because each candidate drug was targeting only one particular MoA ${ }^{25}$. The SARS-CoV2 virus has been selected for method illustration because of the importance of the subject. The virus was introduced into the human population in the Chinese city of Wuhan in the Province of Hubei in December of $2019^{1-4}$. Since then the epidemic of SARS-CoV-2 has rapidly spread Worldwide. The World Health Organization (WHO) has officially declared the SARS-CoV-2 pandemic in March 2020 just three months after its emergence ${ }^{5}$. The novel coronavirus received an official name SARS-CoV-2 and the virus pandemic was called COVID-19 ${ }^{6}$. The formal evaluation and comparison of SARS-CoV-2 drugs can be performed by studying the compound properties by treating patients and performing clinical trials ${ }^{7-10,12}$ or by studying the properties of the corresponding compounds in silico ${ }^{9,11}$ which is done in this work. For method validation we used public ChEMBL (version 28) database ${ }^{26}$ to screen compounds against the most important viral targets, namely 3C-like protease (3CLpro, aka Main protease or Mpro), papain-like protease (PLpro) and RNA-dependent RNA polymerase (RdRp). These targets play a major role in the replication/transcription and host cell recognition and therefore, are vital for the viral reproduction and spread of infection. Because our method doesn't directly use target information but rather analyzes 3D shapes for a compound that was already predicted or experimentally found to be effective against a particular target (we call it a reference compound), one has to choose one (or 
more) such compound(s) as a reference for each target. The focus for each of the above SARSCOV-2 targets (3CLpro, PLpro and RdRp) was on the reference compounds with the highest binding affinities from the recent in silico multi-target repurposing study. ${ }^{24}$

For the new compound search (virtual library screening) we used the same set of reference compounds as we used for the method validation.

\section{METHODS}

Representative conformer space and conformer-by-conformer comparison. The proposed computational algorithm extends the currently available methods ${ }^{20-23}$ and introduces additional search flexibility via the use of the compound conformers. The proposal is to compare multiple possible shapes, adopted via varying environmental conditions, of the same molecule (i.e., conformers) rather than just a single shape that was used before. In particular, the suggested approach is based on the matching of ligand-ligand fingerprints without explicitly using target structure information unlike docking and molecular dynamics approaches that simulate physical binding of a ligand to the target. The supporting theory behind the method is based on the decision to treat conformers, which might have different binding characteristics and properties, as independent entities. In such an approach each conformer has the corresponding independent alignment-free 3D-similarity scoring using the known multi-references. All conformers were generated using the ETKDG algorithm implemented in RDkit ${ }^{27}$. Benchmarking studies have

found ETKDG to be the best-performing freely available conformer generator up-to-date ${ }^{28,29}$ providing diverse and chemically-meaningful conformers reproducing crystal conformations. 
129 Unlike what the majority of computational methods had assumed a couple of decades or so ago 130 (e.g. in the CoMFA method ${ }^{58}$ ), recent research indicates that the bioactive conformation is not 131 necessarily the lowest-energy conformation in the presence of the receptor ${ }^{59-61}$. In particular, as 132 long as an increase in energy for less favorable conformation is compensated by its binding to the 133 target, i.e. the total ligand-target energy is lower than the sum of the energies for the non-bound 134 target and ligand, the bound state is favored. The proposed method emphasizes and relies on this 135 ligand's ability to use its potentially higher energy conformations depending on the target it 136 attempts to bind. Note, however, that when a sufficiently large number of conformers is requested, 137 ETKDG algorithm generates more conformers with lower energy than with higher energy ${ }^{27,28}$, 138 therefore when averaged over all conformers (and we generate 100 conformers per molecule), 139 conformers with the lower energy will contribute more to the total overlap.

140 Actually, one of the things that distinguishes ligand-based 3D virtual screening methods from 2D 141 methods is that one has to start worrying about how many conformers to include in the reference 142 set. If the molecule is flexible, it can assume many shapes and pharmacophores. How to deal with 143 this is one of the fundamental questions in ligand-based virtual screening (LBVS).

144 In a recent paper by Schrödinger team ${ }^{30}$ Cappel et al. performed comprehensive benchmark 145 analysis and found that the number of conformers needed for 3D LBVS is actually relatively low: 146100 or less to achieve good performance. Thus, we used ETKDG to generate 100 conformers per 147 molecule in this work.

148 The authors have called the approach MultiRef3D to emphasize that it is a fast, alignment-free 149 multi-objective optimization protocol that maximizes the $3 \mathrm{D}$ overlap of a query molecule's 150 conformational ensemble with conformational ensembles of multiple reference ligands. The 
151 diagram of the proposed method is summarized in Fig. 1. The formal details of the approach are 152 discussed further.

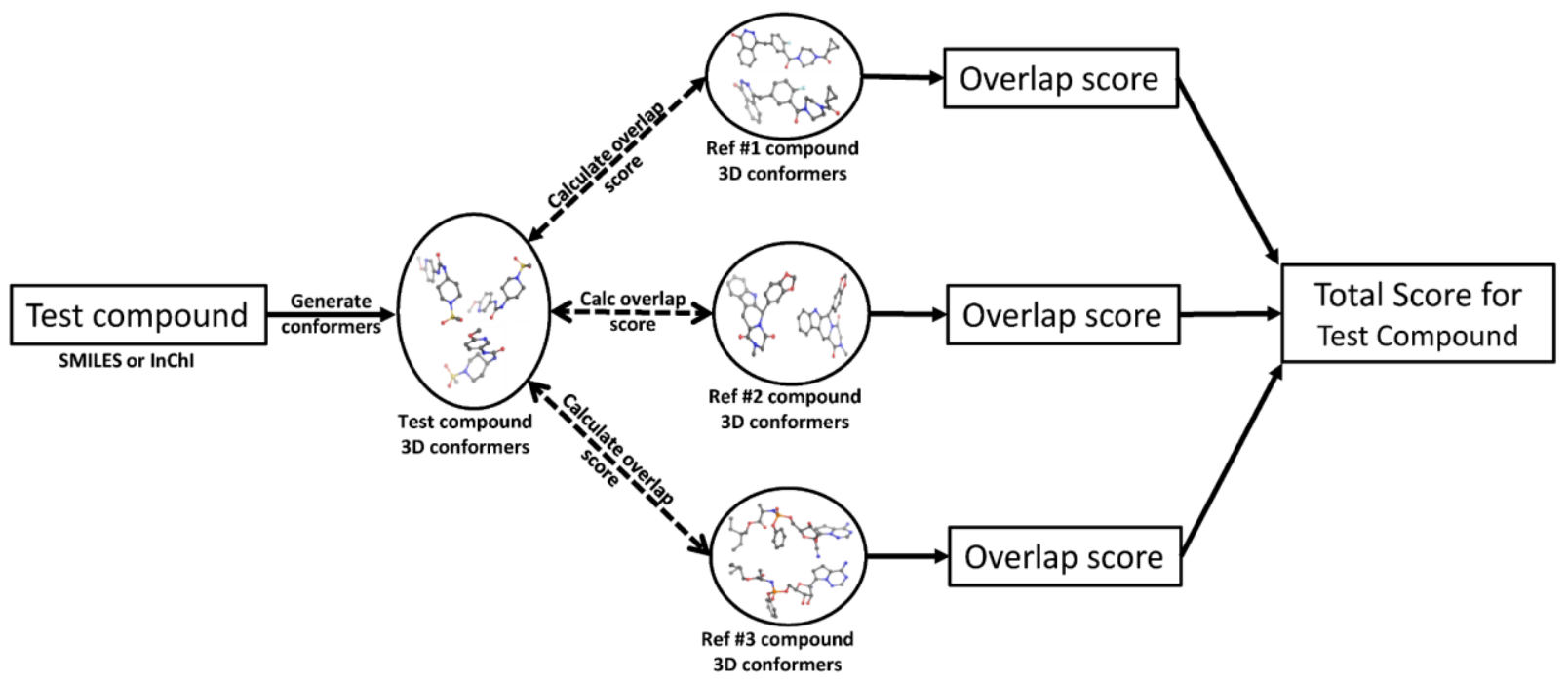

154 Fig. 1. MultiRef3D screening method diagram for multi-conformer and multi-reference screening 155 procedure. For each test compound multiple conformers and the corresponding overlapping scores 156 are computed. Later, the overlapping scores are summed into the total score for the selected test 157 compound. Figure has been created in Microsoft PowerPoint 2016, pyMOL v2.5 (pymol.org) and 158 RDKit v2021.03.01 software (rdkit.org).

Efficiency and a conformer scoring. In the algorithm, each conformation is treated as an independent entity and is characterized by a vector of features (fingerprint) which describes its 3D

162 shape along with the distribution of electrostatic charge (both denoted further as electroshape) 163 across its molecular surface. In this work we used 15-dimensional USRCAT fingerprints ${ }^{31}$ which 164 distil molecular shape into a rotation-invariant descriptor vector made up of 15 real numbers 165 describing distance distribution among atoms, atomic partial charges and atom types. USRCAT 
166 fingerprints were shown to significantly outperform just shape-based fingerprints in recent 167 benchmark tests ${ }^{31,32}$. Since USRCAT fingerprints reflect both relative 3D positions for all atom 168 types and molecular surface charges for each query molecule conformer as well as for all 169 conformers of the reference compound they are very well-suited for alignment-free fast 170 computation of conformer similarity. Each conformer is coded within the algorithm by a single 171 fingerprint represented as a fixed-length vector of numbers which ensures computational 172 efficiency. These fingerprints for each of the query and reference molecule conformers are 173 individually scored by Euclidean distance serving as a similarity measure between two conformers.

174 The Euclidean distance can be viewed as an extension of the Tanimoto similarity measure for non175 binary fingerprints. The fingerprinting of individual conformers for alignment-free comparisons 176 became popular in the past couple of years ${ }^{23,33-35}$ so the proposed method is built on those.

177 Objective Function Optimization. The sum of the conformer-to-conformer similarity scores 178 between the query and a reference compound are compared via an objective similarity function $W_{c}$ 179 for each reference compound $c$. The goal is to maximize the sum of those individual objective 180 similarity functions across all reference compounds of interest $c=1,2, \ldots, C$ where $c$ is a summation 181 index for the desired set of reference compounds:

$$
W_{A l l}=\sum_{c=1}^{C} W_{c}=\sum_{c=1}^{C} \sum_{q=1}^{Q} \sum_{r=1}^{R} S_{q, r}^{(c)}
$$

183 In formula (1) the summand $S_{q, r}^{(c)}$ is the similarity (overlap) of the query conformer $q(q=1,2, \ldots, Q)$ 184 with the conformer $r(r=1,2, \ldots, R)$ for each reference compound $c(c=1,2, \ldots, C)$. For the real-valued 185 fingerprints, the similarity summand between the pair of conformers of interest indexed by query 186 index $q$ and reference index $r$ for compound $c$ is calculated as: 


$$
S_{q, r}^{(c)}=1-(1 / N) \sqrt{\sum_{n=1}^{N}\left(x_{q, n}^{(c)}-x_{r, n}^{(c)}\right)^{2}}
$$

188 where $x_{q, n}^{(c)}$ and $x_{r, n}^{(c)}$ are the corresponding normalized fingerprint vector coordinates for 189 $n=1,2, \ldots, N$. The length (the number of coordinates) of the fingerprint $N$ is determined based on 190 the problem-specific target-ligand interaction characteristics. Since the fingerprint coordinates $x_{q, n}^{(c)}$ 191 and $x_{r, n}^{(c)}$ are normalized (i.e. have values between 0 and 1 for each coordinate $n$ ) the resulting 192 overlap $S_{q, r}^{(c)}$ is maximized with the value equal to 1 when the fingerprints of both conformers are 193 identical and can take the smallest value equal to 0 when all the fingerprint coordinates have a 194 difference equal to 1 i.e. as different as possible at the normalized scale.

195 When the objective is to identify a novel compound for just a single active conformation $(r=1)$ of 196 one $(c=1)$ reference compound (e.g. a reference ligand co-crystallized with one particular target) 197 then all conformers for the query molecule are scored against only one active reference conformer. 198 However, in the case when multiple reference compounds are bound to the same target (or sets of 199 reference compounds bound to multiple targets), the total objective function comes into play. It is 200 important to point out that the proposed method is not limited to the structure-based design 201 situations: when several reference compounds are found to be active in a functional assay (and 202 either the target(s) is unknown or the crystal structure of the target is not available) - the formula 203 works just as well (as long as the ligand structure is known). The method becomes especially 204 handy, when there is a great diversity among active reference compounds, whether the target 205 structural information is known or not - the objective function will extract and sum up the 206 similarities for all of the relevant parts of the fingerprinted conformer representations responsible 207 for the observed activity. 
The query compound can be evaluated against multiple reference compounds on a conformer-byconformer basis. In such a case, the corresponding similarity scores are summed and constitute the multi-reference conformer-level objective function to maximize. This can be readily used in a typical ligand-based design setting. However, instead of just searching for a shape analog of one of the conformers of a reference compound, in the case of multiple references, the algorithm performs a search for such a compound in the virtual library whose conformers have overlapped with conformers of each of those reference compounds. The latter will increase the chances that the selected virtual compound binds the same way to the corresponding targets of each of the references (i.e. the selected compound is capable of forming conformations that resemble active conformations responsible for the MoA of each of the references).

Method validation for known targets. To validate the proposed methodology for the multitarget-specific conformer similarity the three following targets have been used: 3CLpro (Mpro), PLpro, and RdRp. The spike protein has not been included as the validation target since the pharmacological activity may not be correlated directly with the binding affinity to the interfacial site $^{24}$. ChEMBL (version 28) public database ${ }^{26}$ has been chosen as the universe for screening. The selected ChEMBL compounds were already marketed drugs for which at least one target is known. The corresponding ChEMBL extraction query is provided in the manuscript Supplement at GitHub (https:/github.com/quantori/MultiRef3D). The screened set had a total of 2,604 compounds. The corresponding reference compounds for validation were selected from the recent multi-target in silico repurposing study ${ }^{24}$ based on the highest binding affinities for each of the SARS-COV-2 three targets 3CLpro, PLpro and RdRp.

Compounds search based on reference compounds' conformers. One hundred conformers for each of the reference molecules were generated at the MMFF94 level of theory ${ }^{40}$ and each 
231 conformer was ODDT-fingerprinted ${ }^{20}$ and saved in the MongoDB database ${ }^{41}$. The ODDT 232 implementation ${ }^{20}$ of ElectroShape fingerprints ${ }^{22}$ has been selected to demonstrate the proposed 233 approach because these fingerprints are considered to be state-of-the-art in ligand-based virtual 234 screening experiments ${ }^{32,42}$, and they are not limited to binary values.

235 Virtual libraries for screening. Virtual libraries (query compounds) for screening consisted of 236 Enamine ${ }^{41}$ focused "antiviral-like" set (3,995 compounds) and diverse Discovery Diversity Set $237(10,559 \text { compounds })^{44}$. Molecules from each virtual library were simultaneously evaluated against 238 several reference drugs with different MoA (3CLpro, PLpro and RdRp inhibition). A query 239 molecule for which some of its conformers are similar in shape with conformers for all the 240 reference drugs would receive a higher score. In this approach, multiple virtual compounds can be 241 identified to have a good conformer overlap with conformers of the reference drugs.

\section{RESULTS}

244 Method Validation for SARS-CoV-2 Compounds. The highest affinity binder Olaparib (-9.2 $245 \mathrm{kcal} / \mathrm{mol})$ has been selected as a reference compound for 3CLpro, Tadalafil $(-9.2 \mathrm{kcal} / \mathrm{mol})$ for 246 PLpro and Lumacaftor $(-9.9 \mathrm{kcal} / \mathrm{mol})$ for RdRp. However, when multi-target scoring against 247 these three references has been performed, the top ten scoring compounds from ChEMBL had no 248 conformers similar in 3D shape (Euclidean distance < 0.5) to Lumacaftor conformers. Therefore, 249 the Lumacaftor reference has been replaced with the next best in silico RdRp 250 binder ${ }^{24}$ Ergotamine24 (-9.4 kcal/mol binding affinity to RdRp). The resulted scores produced by 251 the proposed method are summarized in Tab. 1: 
255 Tab. 1. Top ten scoring compounds showing simultaneous conformer similarity with the reference 256 compounds Olaparib, Tadalafil, and Ergotamine.

\begin{tabular}{|c|c|c|c|c|c|}
\hline Compound ID & Compound Name & TotalScore & Olaparib & Tadalafil & Ergotamine \\
\hline CHEMBL779 & Tadalafil & 228.46 & 70.70 & 100.00 & 57.76 \\
\hline CHEMBL1737 & Sildenafil citrate & 225.15 & 81.30 & 58.34 & 85.50 \\
\hline CHEMBL521686 & Olaparib & 223.08 & 100.00 & 57.61 & 65.48 \\
\hline CHEMBL105442 & $\mathrm{Ci}-1040$ & 220.40 & 80.68 & 79.16 & 60.56 \\
\hline CHEMBL129857 & As-602868 & 220.16 & 78.27 & 74.50 & 67.39 \\
\hline CHEMBL2037511 & Epelsiban & 219.86 & 81.58 & 70.28 & 68.01 \\
\hline CHEMBL565612 & Sotrastaurin & 219.13 & 79.93 & 69.36 & 69.83 \\
\hline CHEMBL1516474 & Tegaserod maleate & 217.83 & 80.22 & 76.56 & 61.05 \\
\hline CHEMBL1236682 & Refametinib & 217.78 & 76.01 & 81.57 & 60.20 \\
\hline CHEMBL1923502 & $\begin{array}{l}\text { Ulimorelin } \\
\text { hydrochloride }\end{array}$ & 217.56 & 76.29 & 74.79 & 66.47 \\
\hline
\end{tabular}

258 Both Olaparib and Tadalafil had the highest scores which confirmed the previous finding ${ }^{24}$ that 259 these compounds are simultaneously good binders for both 3CLpro and PLpro. Our method has 260 also picked up Sildenafil (more commonly known under the brand name Viagra) which just like 261 Tadalafil (aka Cialis) is also known as a classical PDE5A inhibitor. Although those compounds 262 are predominantly used in the treatment of male erectile dysfunction and pulmonary hypertension, 
263 it was shown ${ }^{45}$ that in the presence of SARS-COV-2 infection, PDE5 inhibitors prevent 264 thromboembolism caused by inflammatory processes in COVID-19 patients via NO/cGMP 265 pathway and are potent inhibitors of 3CLpro ${ }^{46}$

266 Ci-1040 and Refametinib are the other two hits from Tab. 1 and are potent MEK inhibitors with 267 high 3D shape similarity to both Olaparib and Tadalafil. MEK inhibitors, including Olaparib ${ }^{47}$ 268 were recently demonstrated to reduce cellular expression of ACE2 while stimulating NK-mediated 269 cytotoxicity and attenuating inflammatory cytokines during the severe stage of SARS-CoV-2 270 infection ${ }^{48}$. Ci-1040 was also previously shown to display a broad anti-influenza virus activity in 271 vitro and to provide a prolonged treatment window compared to the standard of care in vivo, 272 specifically in lung cells $\mathrm{s}^{49}$.

273 The other hit from Tab. 1 is Sotrastaurin which is a PKC inhibitor and has been experimentally 274 shown to inhibit SARS-COV-2 replication in vivo ${ }^{50}$ and has been found to be among the best 275 3CLpro binders during in silico ZINC database screening study ${ }^{51}$ Yet another notable hit among 276 the top ten selected compounds in Tab. 1 is As-602868: a potent IKK2 inhibitor. This class of 277 compounds is currently preclinically tested for NF-kB mediated cytokine storm attenuation in 278 severe COVID-19 patients ${ }^{52}$.

279 The other top hit, Epelsiban, was originally developed as an oxytocin receptor agonist. However, 280 it has been recently shown ${ }^{54}$ that oxytocin plays a major role in activation of NF-kB-mediated 281 pathways. Interestingly, recent research has revealed 50 that Remdesivir (in addition to being a 282 potent $\mathrm{RdRp}$ inhibitor) is also reducing viral replication via NF-kB pathway. Therefore, this hit 283 serves as an example of non-obvious 3D-shape-based drug repurposing idea generation linked to 284 the relevant yet non-primary SARS-COV-2 inhibiting mechanisms of reference compounds. 
285 Thus in our second validation experiment the RdRp reference compound Ergotamine has been 286 replaced with Remdesivir which, as we already mentioned, is a well-established RdRp inhibitor 287 and cytokine storm attenuator that works via NF-kB pathway. The resulted scores produced in the 288 second scoring setup are summarized in Tab. 2:

289 Tab. 2. Top ten scoring compounds showing simultaneous conformer similarity with the reference 290 compounds Olaparib, Tadalafil, and Remdesivir.

\begin{tabular}{|c|c|c|c|c|c|}
\hline Compound ID & Compound Name & TotalScore & Olaparib & Tadalafil & Remdesivir \\
\hline CHEMBL1694 & $\begin{array}{l}\text { Benazepril } \\
\text { hydrochloride }\end{array}$ & 180.82 & 66.67 & 64.26 & 49.89 \\
\hline CHEMBL515606 & Cilazapril & 180.61 & 64.56 & 61.56 & 54.50 \\
\hline CHEMBL495727 & At-9283 & 179.03 & 68.17 & 56.15 & 54.71 \\
\hline CHEMBL2107495 & $\begin{array}{l}\text { Temafloxacin } \\
\text { hydrochloride }\end{array}$ & 178.94 & 67.15 & 55.78 & 56.01 \\
\hline CHEMBL1200779 & $\begin{array}{l}\text { Trovafloxacin } \\
\text { mesylate }\end{array}$ & 178.60 & 66.24 & 54.02 & 58.35 \\
\hline CHEMBL340978 & Benoxaprofen & 178.27 & 68.56 & 56.54 & 53.16 \\
\hline CHEMBL8 & Ciprofloxacin & 177.05 & 63.21 & 57.19 & 56.65 \\
\hline CHEMBL1200831 & $\begin{array}{l}\text { Spirapril } \\
\text { hydrochloride }\end{array}$ & 177.00 & 65.28 & 60.24 & 51.47 \\
\hline CHEMBL1201011 & $\begin{array}{l}\text { Quinapril } \\
\text { hydrochloride }\end{array}$ & 176.84 & 66.32 & 60.40 & 50.13 \\
\hline CHEMBL1168 & Ramipril & 176.54 & 65.32 & 63.26 & 47.96 \\
\hline
\end{tabular}


292 For Olaparib, Tadalafil, and Remdesivir reference compounds half of the top ten hits 293 (Benoxaprofen, Ciprofloxacin, Spirapril hydrochloride, Quinapril hydrochloride and Ramipril) 294 turned out to be ACE inhibitors and coagulation modifiers acting via NF-kB related pathways ${ }^{55,56}$ ! 295 In addition, all of them turned out to be also good binders of 3CLpro ${ }^{57}$.

296 The other hits were Temafloxacin and Trovafloxacin, predicted to be potent 3CLpro ligands ${ }^{55}$ and 297 experimentally shown to inhibit virus replication ${ }^{56,57}$, and anti-inflammatory drugs Benoxaprofen 298 and Ciproflaxin predicted to target 3CLpro ${ }^{58,59}$ as well.

299 Concluding the list is an interesting multi-target Aurora/JAK inhibitor hit: compound At-9283. 300 JAK inhibitors, in general, have promising therapeutic potential for SARS-COV-2 treatment with 301 their dual anti-inflammatory and anti-viral effects ${ }^{60}$. At-9283, however, has also been recently 302 identified to reverse SARS-COV-2 transcriptomic signature ${ }^{61}$ and due to its matching tipiracil's 303 3D pharmacophore scaffold also inhibits SARS-COV-2 Nsp15 endoribonuclease ${ }^{62,63}$ and targets 304 3CLpro as well ${ }^{64,65}$.

305 Virtual library screening for multi-target SARS-CoV-2 compounds. The results from the 306 focused ("antiviral-like") and diverse ("Discovery Diversity Set") are summarized in Tab 1 and 2 307 respectively. . The algorithm visual summary is displayed in Fig. 1 for the $W_{A l l}$ objective function. 308 Tables 3 and 4 summarize the direct application results of the Enamine ${ }^{41}$ focused "antiviral-like" 309 and "Diverse Discovery Set" virtual library screening. The first two columns of the Tables contain 310 query compound IDs and their computed overlap scores. The rows are sorted according to the total 311 sum overlap score displayed in the second column. 
313 Tab. 3. The top scoring compounds from the Enamine "antiviral-like" virtual library (the first

314 column) are sorted by their total overlap score $W_{A l l}$ (the second column). The values in the other

315 columns correspond to the sums of the overlap scores of the conformers for the corresponding

316 reference compounds.

\begin{tabular}{|c|c|c|c|c|c|}
\hline Compound ID & Wall & Olaparib & Tadalafil & Ergotamine & Remdesivir \\
\hline Z1693453146 & 254.11 & 71.68 & 56.30 & 76.76 & 49.38 \\
\hline Z434669842 & 248.84 & 66.18 & 55.33 & 69.92 & 57.41 \\
\hline Z1381427631 & 248.57 & 66.63 & 52.86 & 72.84 & 56.24 \\
\hline Z1381425049 & 247.97 & 66.09 & 53.03 & 71.98 & 56.88 \\
\hline Z1313285936 & 246.97 & 67.70 & 55.51 & 72.23 & 51.54 \\
\hline Z826278840 & 246.37 & 65.61 & 56.67 & 68.79 & 55.30 \\
\hline Z94559538 & 245.69 & 70.41 & 55.41 & 65.24 & 54.64 \\
\hline Z435640438 & 245.21 & 63.85 & 54.62 & 72.45 & 54.29 \\
\hline Z435642248 & 245.13 & 64.04 & 55.31 & 71.85 & 53.93 \\
\hline Z827564114 & 244.89 & 64.93 & 57.02 & 70.19 & 52.75 \\
\hline
\end{tabular}

317

318

319

320

321 
322 Tab. 4. The top scoring query compounds from the Enamine a "Diverse Discovery Set" virtual 323 library (the first column) are sorted by their total overlap score $W_{\text {All }}$ (the second column). The

324 values in the other columns correspond to the sums of the overlap scores of the conformers for the 325 corresponding reference compounds.

\begin{tabular}{|c|c|c|c|c|c|}
\hline Compound ID & Wall & Olaparib & Tadalafil & Ergotamine & Remdesivir \\
\hline Z1760146546 & 255.19 & 74.41 & 60.57 & 74.94 & 45.27 \\
\hline Z3077896041 & 254.26 & 67.67 & 57.38 & 75.53 & 53.67 \\
\hline Z2911083836 & 253.23 & 72.49 & 58.63 & 75.85 & 46.27 \\
\hline Z2446617864 & 252.49 & 73.18 & 59.52 & 75.06 & 44.72 \\
\hline Z1139281415 & 252.30 & 69.01 & 57.39 & 75.88 & 50.02 \\
\hline Z1354703942 & 251.79 & 77.22 & 61.63 & 80.63 & 32.32 \\
\hline Z2256366543 & 251.27 & 66.54 & 54.27 & 74.18 & 56.28 \\
\hline Z1139281396 & 250.99 & 68.40 & 57.90 & 74.43 & 50.27 \\
\hline Z1139280685 & 250.51 & 68.34 & 57.88 & 74.50 & 49.79 \\
\hline Z2959367287 & 250.32 & 69.54 & 55.40 & 72.80 & 52.58 \\
\hline
\end{tabular}

327 For the visual illustration of the algorithm results two compounds with the highest scores from

328 Tab. 3 and 4 have been presented in Fig. 2, panels A and B respectively. It is worth noting that

329 these compounds are quite flexible molecules due to their amidebridge around which the ring 330 substructures can rotate, which ensures the ability of those molecules to accommodate different 331 targets. 

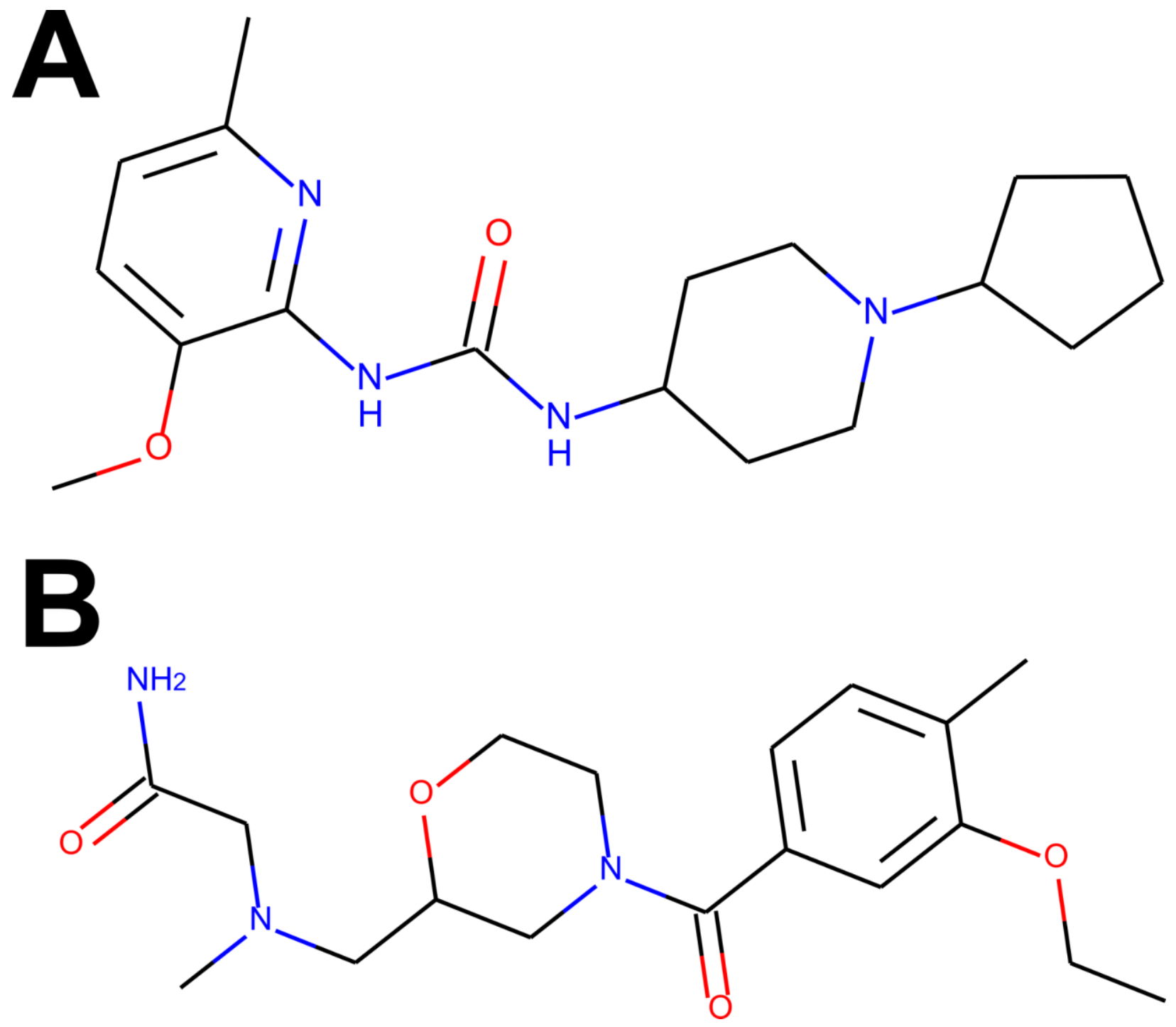

337 Fig. 2. The compounds presented in panels A and B are the top hits Z1693453146 (Wall=254.11) and ZZ1760146546 (Wall $=255.19)$ from the non-overlapping "antiviral-like" and "Discovery Diversity" libraries respectively. One can immediately observe, however, that the compounds share a lot of similarity, in particular overall shape and amide bridge connecting heterocycles. The 
341 bridge allows for 3D flexibility for the molecule to change conformation and bind to multiple 342 targets. Figure has been created in Microsoft PowerPoint 2016, pyMOL v2.5 (pymol.org) and 343 RDKit v2021.03.01 software (rdkit.org).

344 Fig. 3 demonstrates how the best-matching conformers of the top hit Z1693453146 spatially align 345 with the active conformation for each reference drug. One can observe that the majority of 346 hydrogen donors and acceptors from the top hit conformer and reference conformers are aligned

347 very well mimicking the interaction patterns with each target. At least partial spatial alignment of 348 atom types is expected from the top hit conformers since atom types as well as their relative 3D 349 positions is the essence of the USRCAT fingerprints ${ }^{31}$.

A

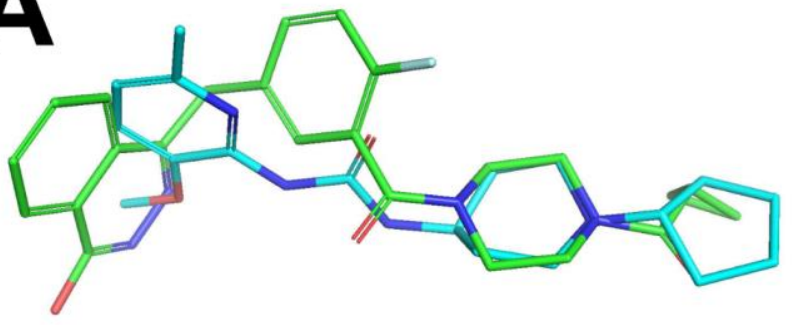

B

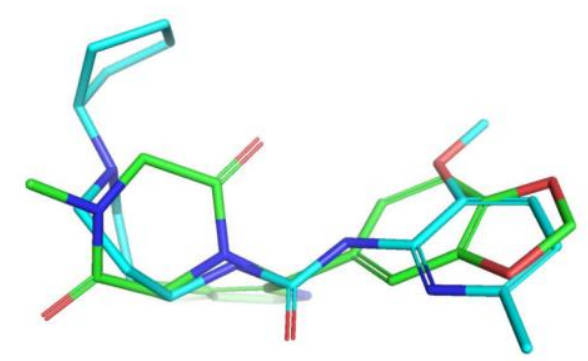

0

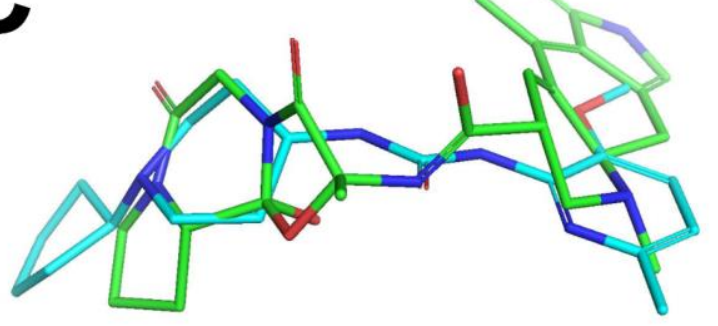

D

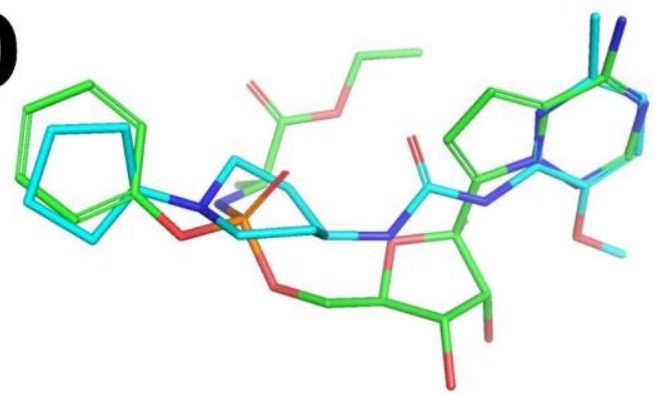

351

352 Fig. 3. Active conformers of the Reference compounds (Olaparib, Tadalafil, Ergotamine and

353 Remdesivir on panels A, B, C and D respectively) aligned with the best matching conformers of the top hit Z1693453146 (Wall = 254.11). Carbon-carbon bonds for the reference compounds and 
355 the tTop hHit are shown in green and cyan respectively ( $\mathrm{C}-\mathrm{N}$ and $\mathrm{C}-\mathrm{O}$ bonds are conventionally

356 shown in blue and red). Figure has been created in Microsoft PowerPoint 2016, pyMOL v2.5

357 (pymol.org) and RDKit v2021.03.01 software (rdkit.org).

\section{DISCUSSION}

360 Computation efficiency and availability of the method. The proposed method does not rely on 361 laborious docking and molecular dynamics setup, especially in the multi-target case, where target 362 preparation and choice of method i.e. direct docking to a fixed-coordinate target or Molecular 363 Dynamics -based ensemble energy minimization are of utmost importance and require deep 364 expertise. Fingerprint comparison is orders of magnitude faster and simpler (only requires simple 365 structural information in the form of either isomeric SMILES or InChI). The entire setup is 366 presented in our Supplement that can be universally used for any multi-target screening and 367 optimization whenever reference compounds for each of the targets are available. Naturally, 368 further hit refinement (ADMETox, PK/PD, etc) is necessary if the screened universe is not limited 369 to the drugs with the well-known safety profiles.

370 Application for drug-repurposing. Depending on what is known about the indication or 371 marketed drug of interest (targets, MoAs, other existing drugs for the same indication) the 372 proposed methods (or a combination thereof) can be used to find other non-obvious molecules 373 whose shape and the surface electrostatic charge is similar to that of the marketed drug. The 374 methods can also be used to search for the cumulative similarity to conformers of the multiple 375 drugs used to treat this disease indication. 
376 In the proposed approach multiple conformers of the query ligand have been compared with

377 conformers from multiple reference compounds whose therapeutic effect of interest is achieved

378 via different mechanisms of bindings to different targets, e.g. by inhibiting major proteases

3793 CLpro and PLpro ${ }^{63}$ and RNA-dependent RNA polymerase (RdRp) ${ }^{6465,66}$. An "ideal drug” would

380 contain conformers that resemble (as many as possible) conformers of all of the reference drugs,

381 thus increasing chances that the drug inhibits SARS-CoV-2 via multi-MoA routes and is more

382 effective than each individual reference drug.

383 Note on applications for structure-based designs. When the crystal structure of the target protein

384 is known and the reference ligand is co-crystallized in its active conformation (structure-based

385 design), we can use this information about the reference compound and evaluate the query

386 molecules against only one, the active (co-crystallized) reference ligand conformation $\left(r=r_{\text {active }}\right)$

387 in formulas (1) and (2). Confirmation by direct docking for the fingerprint-matched queries can be

388 used to confirm the match.

389 Our methodology emphasizes pursuit of candidate compounds that achieve therapeutic effect (e.g.

390 stops SARS-CoV-2 proliferation) by multiple MoA routes. A successful candidate compound

391 would contain conformers targeting the two major proteases 3CLpro and PLpro RdRpall at the

392 same time by increasing chances that the compound would protect against SARS-CoV-2 much

393 more effectively. Naturally, all successful candidates would need to be further screened and

394 filtered for proper ADME-Tox and other drug-likeness properties. Binding to anti-targets, e.g.

395 hERG, can be explicitly incorporated to this methodology by adding the corresponding terms

396 (similarities to known hERG-binding ligands) to the overlap sum with a negative sign. Even

397 though many computational methods exist to evaluate hERG in particular as well as other common

398 tox liabilities, when an anti-target is very specific and less commonly known as "pure tox target" 
399 (e.g. undesired binding to D2 receptor for many modern CNS drugs), the explicit inclusion of 400 similarity score to such anti-target with a negative sign can greatly streamline the overall drug 401 optimization process.

402

\section{CONCLUSION}

404 We have demonstrated and validated the usefulness of the multi-reference computationally 405 efficient optimization approach in drug discovery screening and repurposing scenarios. The 406 method represents each molecule as an ensemble of flexible conformers that would choose the 407 best possible conformation for each presented target-binding opportunity. Application of this 408 approach to SARS-CoV-2 produced several antiviral drug candidates that are designed to protect 409 against SARS-CoV-2 by multiple mechanisms simultaneously.

\section{LIST OF ABBREVIATIONS}

412 ADME-Tox - Absorption, Distribution, Metabolism, Excretion and Toxicity

413 GPU - Graphics processing unit

414 CNS - Central nervous system

415 CoMFA - Comparative molecular field analysis

416 COVID-19 - Coronavirus Disease of 2019

417 CPU - Central processing unit

418 hERG - Human Ether-a-go-go-related Gene 
$419 \operatorname{MoA}(\mathrm{s})$ - Mechanism of Action(s)

420 ODDT - Open Drug Discovery Toolkit

$421 \quad$ RNA - Ribonucleic acid

422 ROCS - Rapid overlay of chemical structures

423 SARS-CoV-2 - Severe acute respiratory syndrome coronavirus 2

424 WHO - World Health Organization

425

426 DECLARATIONS

427 Availability of data and materials

428 Code that has been used for analysis and for manuscript preparation can be found at Quantori

429 public GitHub repository online ${ }^{67}$. Data (ligand structures) from REAL focused libraries can be 430 downloaded from the Enamine Ltd. website ${ }^{68}$.

431 Competing interests

432 The proposed method has been submitted for a patent. The patent application number is 63061790

433 at the United States Patent and Trademark Office and as of October 17, 2020, the patent is pending.

434 The patent can be a source of financial income for authors Vadim Alexandrov (VA) and Yuriy

435 Gankin (YG). 
439 The author Vadim Alexandrov (VA), a founder of a consulting company Liquid Algo LLC in

440 Hopewell Junction, New York, United States received no compensation for this work. The author 441 Yuriy Gankin (YG) is employed by the commercial company Quantori in Cambridge,

442 Massachusetts, United States. Alexander Kirpich (AK) is employed at the School of Public Health 443 at a non-profit institution Georgia State University. Quantori provided support in the form of salary 444 and relevant publication and patent fees for YG. YG received no compensation for this work. AK 445 received no funding or any other financial support for this project.

\section{Authors' contributions}

447 Vadim Alexandrov (VA), Alexander Kirpich (AK), and Yuriy Gankin (YG) are the authors of the 448 manuscript. VA and YG proposed the manuscript idea, obtained the data, implemented routine 449 coding operations, and wrote the preliminary version of the manuscript. AK performed an 450 additional literature review and wrote the final version of the manuscript. YG also provided the 451 overall guidance for the project and participated in the manuscript preparation.

\section{Acknowledgments}

453 The authors would like to acknowledge Nika Tsutskiridze and Daviti Khatchilava for their 454 assistance in extracting information from clinical trials and peer reviewed literature. The authors 455 also want to acknowledge Alexander Proutsky and John Reynders for their suggestions and 456 comments during the manuscript preparation.

457 Authors' information 
458 VA holds a Ph.D. in Computational Chemistry from The University of Arizona and a Ph.D. in 459 BioInformatics from Yale University. He has over 20 years of experience in computer-aided drug 460 design and pioneered hit generation and lead optimization paradigm in high-throughput in-vivo 461 phenotypic drug discovery.

462 AK is a biostatistician and an assistant professor in the Department of Population Health Sciences 463 at the School of Public Health at Georgia State University. AK holds a Ph.D. in biostatistics from 464 the University of Florida and has expertise in infectious disease statistical modeling (cholera, 465 dengue, HIV, anthrax, and others), epidemiology, and bioinformatics. The interests and goals of 466 AK are broad and target application of statistical methods to public health and biomedical research 467 questions and policies and to address statistical challenges, such as missing data, asymptomatic 468 infections, underreporting, and noise during data acquisition.

469 YG serves as Chief Scientific Officer at Quantori, a developer of intelligent IT and data science 470 solutions for life science, pharma, and healthcare organizations. Yuriy's expertise is in analytical 471 chemistry, data science, and chem- and bioinformatics. He holds a Ph.D. in Analytical Chemistry 472 from Tufts University and an MBA from the Massachusetts Institute of Technology Sloan School 473 of Management. 


\section{REFERENCES}

481 1. Zhu, N. et al. A Novel Coronavirus from Patients with Pneumonia in China, 2019. N. Engl.

482 J. Med. 382, 727-733 (2020).

2. Lu, H., Stratton, C. W. \& Tang, Y.-W. Outbreak of pneumonia of unknown etiology in Wuhan, China: The mystery and the miracle. J. Med. Virol. 92, 401-402 (2020).

3. Huang, C. et al. Clinical features of patients infected with 2019 novel coronavirus in 486 Wuhan, China. The Lancet vol. 395 497-506 (2020).

487 488

489

490

491

4. Chen, N. et al. Epidemiological and clinical characteristics of 99 cases of 2019 novel coronavirus pneumonia in Wuhan, China: a descriptive study. The Lancet vol. 395 507-513 (2020).

5. WHO Director-General's opening remarks at the media briefing on COVID-19 - 11 March 2020. https://www.who.int/dg/speeches/detail/who-director-general-s-opening-remarks-at492 the-media-briefing-on-COVID-19---11-march-2020.

493 6. Naming the coronavirus disease (COVID-19) and the virus that causes it.

494 https://www.who.int/emergencies/diseases/novel-coronavirus-2019/technical495 guidance/naming-the-coronavirus-disease-(covid-2019)-and-the-virus-that-causes-it.

496 7. Cascella, M., Rajnik, M., Cuomo, A., Dulebohn, S. C. \& Di Napoli, R. Features, 497 Evaluation, and Treatment of Coronavirus (COVID-19). in StatPearls [Internet] (StatPearls $498 \quad$ Publishing, 2020).

499 8. Sanders, J. M., Monogue, M. L., Jodlowski, T. Z. \& Cutrell, J. B. Pharmacologic 500 Treatments for Coronavirus Disease 2019 (COVID-19): A Review. JAMA (2020) 501 doi:10.1001/jama.2020.6019.

502 9. Elmezayen, A. D., Al-Obaidi, A., Şahin, A. T. \& Yelekçi, K. Drug repurposing for 
coronavirus (COVID-19): screening of known drugs against coronavirus 3CL hydrolase and protease enzymes. J. Biomol. Struct. Dyn. 1-13 (2020).

10. Zhang, W. et al. The use of anti-inflammatory drugs in the treatment of people with severe coronavirus disease 2019 (COVID-19): The experience of clinical immunologists from China. Clin. Immunol. 108393 (2020).

11. Beck, B. R., Shin, B., Choi, Y., Park, S. \& Kang, K. Predicting commercially available antiviral drugs that may act on the novel coronavirus (SARS-CoV-2) through a drug-target interaction deep learning model. Comput. Struct. Biotechnol. J. 18, 784-790 (2020).

12. Sargiacomo, C., Sotgia, F. \& Lisanti, M. P. COVID-19 and chronological aging: senolytics and other anti-aging drugs for the treatment or prevention of corona virus infection? Aging 12, 6511-6517 (2020).

13. Rocs, O. E. OpenEye Scientific Software, Inc., Santa Fe, NM, USA. (2008).

14. Hawkins, P. C. D., Skillman, A. G. \& Nicholls, A. Comparison of shape-matching and docking as virtual screening tools. J. Med. Chem. 50, 74-82 (2007).

15. Venhorst, J., Núñez, S., Terpstra, J. W. \& Kruse, C. G. Assessment of scaffold hopping efficiency by use of molecular interaction fingerprints. J. Med. Chem. 51, 3222-3229 (2008).

16. Sheridan, R. P., McGaughey, G. B. \& Cornell, W. D. Multiple protein structures and multiple ligands: effects on the apparent goodness of virtual screening results. J. Comput. Aided Mol. Des. 22, 257-265 (2008).

17. Rush, T. S., 3rd, Grant, J. A., Mosyak, L. \& Nicholls, A. A shape-based 3-D scaffold hopping method and its application to a bacterial protein-protein interaction. J. Med. Chem. 48, 1489-1495 (2005). 
18. Haque, I. S. \& Pande, V. S. PAPER--accelerating parallel evaluations of ROCS. J. Comput. Chem. 31, 117-132 (2010).

19. FastROCS. OpenEye Scientific Software, Inc., Santa Fe, NM, USA. (2011).

20. Wójcikowski, M., Kukiełka, M., Stepniewska-Dziubinska, M. M. \& Siedlecki, P. Development of a protein-ligand extended connectivity (PLEC) fingerprint and its application for binding affinity predictions. Bioinformatics 35, 1334-1341 (2019).

21. Ballester, P. J. \& Richards, W. G. Ultrafast shape recognition to search compound databases for similar molecular shapes. J. Comput. Chem. 28, 1711-1723 (2007).

22. Armstrong, M. S. et al. ElectroShape: fast molecular similarity calculations incorporating shape, chirality and electrostatics. J. Comput. Aided Mol. Des. 24, 789-801 (2010).

23. Axen, S. D. et al. A Simple Representation of Three-Dimensional Molecular Structure. J. Med. Chem. 60, 7393-7409 (2017).

24. Murugan, N. A., Kumar, S., Jeyakanthan, J. \& Srivastava, V. Searching for target-specific and multi-targeting organics for Covid-19 in the Drugbank database with a double scoring approach. Sci. Rep. 10, 1-16 (2020).

25. Recent progress and challenges in drug development against COVID-19 coronavirus (SARS-CoV-2) - an update on the status. Infect. Genet. Evol. 83, 104327 (2020).

26. Gaulton, A. et al. ChEMBL: a large-scale bioactivity database for drug discovery. Nucleic Acids Res. 40, D1100-D1107 (2011).

27. Wang, S., Witek, J., Landrum, G. A. \& Riniker, S. Improving Conformer Generation for Small Rings and Macrocycles Based on Distance Geometry and Experimental TorsionalAngle Preferences. J. Chem. Inf. Model. 60, 2044-2058 (2020).

28. Friedrich, N.-O. et al. High-Quality Dataset of Protein-Bound Ligand Conformations and 
Its Application to Benchmarking Conformer Ensemble Generators. J. Chem. Inf. Model. 57, 529-539 (2017).

29. Friedrich, N.-O. et al. Benchmarking Commercial Conformer Ensemble Generators. J. Chem. Inf. Model. 57, 2719-2728 (2017).

30. Cappel, D., Dixon, S. L., Sherman, W. \& Duan, J. Exploring conformational search protocols for ligand-based virtual screening and 3-D QSAR modeling. J. Comput. Aided Mol. Des. 29, 165-182 (2014).

31. Schreyer, A. M. \& Blundell, T. USRCAT: real-time ultrafast shape recognition with pharmacophoric constraints. J. Cheminform. 4, (2012).

32. Bonanno, E. \& Ebejer, J.-P. Applying Machine Learning to Ultrafast Shape Recognition in Ligand-Based Virtual Screening. Frontiers in Pharmacology vol. 10 (2020).

33. Wójcikowski, M., Zielenkiewicz, P. \& Siedlecki, P. Open Drug Discovery Toolkit (ODDT): a new open-source player in the drug discovery field. J. Cheminform. 7, 26 (2015).

34. Gladysz, R. et al. Spectrophores as one-dimensional descriptors calculated from threedimensional atomic properties: applications ranging from scaffold hopping to multi-target virtual screening. J. Cheminform. 10, 9 (2018).

35. Wang, Y. et al. TF3P: Three-Dimensional Force Fields Fingerprint Learned by Deep Capsular Network. J. Chem. Inf. Model. 60, 2754-2765 (2020).

36. ClinicalTrials.gov. http://ClinicalTrials.gov.

37. Chen, X. \& Geiger, J. D. Janus sword actions of chloroquine and hydroxychloroquine against COVID-19. Cell. Signal. 73, 109706 (2020).

38. Frediansyah, A., Nainu, F., Dhama, K., Mudatsir, M. \& Harapan, H. Remdesivir and its antiviral activity against COVID-19: A systematic review. Clin Epidemiol Glob Health 
(2020) doi:10.1016/j.cegh.2020.07.011.

573 39. Agrawal, U., Raju, R. \& Udwadia, Z. F. Favipiravir: A new and emerging antiviral option in COVID-19. Armed Forces Med. J. India 76, 370-376 (2020).

575 40. Halgren, T. A. Merck molecular force field. I. Basis, form, scope, parameterization, and 576 performance of MMFF94. Journal of Computational Chemistry vol. 17 490-519 (1996).

577 41. MongoDB Atlas Database. https://www.mongodb.com.

578 42. Cortés-Cabrera, A., Morris, G. M., Finn, P. W., Morreale, A. \& Gago, F. Comparison of 579 580

43. http://zinc15.docking.org/. http://zinc15.docking.org/.

44. REAL Database - Enamine. https://enamine.net/library-synthesis/real-compounds/realdatabase.

45. Shirvaliloo, M. Targeting the SARS-CoV-2 3CLpro and NO/cGMP/PDE5 pathway in 585

48. Zhou, L. et al. MEK inhibitors reduce cellular expression of ACE2, pERK, pRb while

47. Vena, F. et al. MEK inhibition leads to BRCA2 downregulation and sensitization to DNA damaging agents in pancreas and ovarian cancer models. Oncotarget 9, 11592-11603 (2018).

46. Jin, Z. et al. Structure of M pro from SARS-CoV-2 and discovery of its inhibitors. Nature 582, 289-293 (2020). 
49. The MEK-inhibitor CI-1040 displays a broad anti-influenza virus activity in vitro and provides a prolonged treatment window compared to standard of care in vivo. Antiviral Res. 142, 178-184 (2017).

50. Liu, S. et al. Potential Antiviral Target for SARS-CoV-2: A Key Early Responsive Kinase during Viral Entry. CCS Chemistry 559-568 (2021) doi:10.31635/ccschem.021.202000603.

51. Olubiyi, O. O., Olagunju, M., Keutmann, M., Loschwitz, J. \& Strodel, B. High Throughput Virtual Screening to Discover Inhibitors of the Main Protease of the Coronavirus SARSCoV-2. Molecules 25, 3193 (2020).

52. Kircheis, R. et al. NF- $\kappa$ B Pathway as a Potential Target for Treatment of Critical Stage COVID-19 Patients. Front. Immunol. 11, (2020).

53. Hariharan, A., Hakeem, A. R., Radhakrishnan, S., Reddy, M. S. \& Rela, M. The Role and Therapeutic Potential of NF-kappa-B Pathway in Severe COVID-19 Patients. Inflammopharmacology 1 .

54. Oxytocin activates NF- $\mathrm{BB}$-mediated inflammatory pathways in human gestational tissues. Mol. Cell. Endocrinol. 403, 64-77 (2015).

55. Hernández-Presa, M. et al. Angiotensin-converting enzyme inhibition prevents arterial nuclear factor-kappa B activation, monocyte chemoattractant protein-1 expression, and macrophage infiltration in a rabbit model of early accelerated atherosclerosis. Circulation 95, (1997).

56. Burzynski, L. C. et al. The Coagulation and Immune Systems Are Directly Linked through the Activation of Interleukin-1 $\alpha$ by Thrombin. Immunity vol. 50 1033-1042.e6 (2019).

57. Ísis Venturi Biembengut, T. de A. C. B. de S. Coagulation modifiers targeting SARS-CoV2 main protease Mpro for COVID-19 treatment: an in silico approach. Mem. Inst. Oswaldo 
Cruz 115, (2020).

619 58. Optibrium. Optibrium - StarDrop: Nova - A new generation of possibilities.

620 https://www.optibrium.com/stardrop/stardrop-nova.php.

621 59. Gohda, K., Mori, I., Ohta, D. \& Kikuchi, T. 10.1023/A:1008193217627. Journal of

622 Computer-Aided Molecular Design vol. 14 265-275 (2000).

623 60. Mackerell, A. D., Jr. Empirical force fields for biological macromolecules: overview and 624 issues. J. Comput. Chem. 25, 1584-1604 (2004).

625

626

627

628

629

630

631

632

633

634

635

636

637

638

639

640

61. Hasegawa, K., Arakawa, M. \& Funatsu, K. Rational choice of bioactive conformations through use of conformation analysis and 3-way partial least squares modeling. Chemometrics Intellig. Lab. Syst. 50, 253-261 (2000).

62. Acharya, C., Coop, A., Polli, J. E. \& Mackerell, A. D., Jr. Recent advances in ligand-based drug design: relevance and utility of the conformationally sampled pharmacophore approach. Curr. Comput. Aided Drug Des. 7, 10-22 (2011).

63. Ullrich, S. \& Nitsche, C. The SARS-CoV-2 main protease as drug target. Bioorganic \& Medicinal Chemistry Letters vol. 30127377 (2020).

64. Elfiky, A. A. SARS-CoV-2 RNA dependent RNA polymerase (RdRp) targeting: an perspective. J. Biomol. Struct. Dyn. 1-9 (2020).

65. Li, W. et al. Angiotensin-converting enzyme 2 is a functional receptor for the SARS coronavirus. Nature vol. 426 450-454 (2003).

66. Vincent, M. J. et al. Chloroquine is a potent inhibitor of SARS coronavirus infection and spread. Virol. J. 2, 69 (2005).

67. Website. github.com - Quantori repository https://github.com/quantori/MultiRef3D.

68. Targeted Libraries. https://enamine.net/hit-finding/focused-libraries/. 
641 